\title{
modelo lógico de la supervisión como actividad educativa para la formación en psicología clínica: concepciones de supervisores clínicos
}

\author{
Logic Model of Supervision as an Educational fictivity for Training \\ in Clinical Psychology: Conceptions of Clinical Supervisors \\ modelo lógico da supervisão como atividade educativa para a formação \\ em psicologia clínica: conceições de superuisores clínicos
}

\author{
Hamer Bastidas-Bilbao*, Ana María Velásquez* \\ Universidad de los Andes, Bogotá, Colombia.
}

Doi: http://dx.doi.org/10.12804/ap134.2.2016.06

\section{Resumen}

En este estudio cualitativo se derivó un modelo lógico de la supervisión clínica como actividad educativa programática. Se efectuó una entrevista semiestructurada a diez supervisores de los programas de pregrado y maestría profesionalizante ofrecidos por una universidad privada en Bogotá, Colombia. Las respuestas se analizaron siguiendo el método NCT (en inglés, noticing things, collecting things, thinking about things). Además de los descriptores de insumos, actividades y resultados, también se identificaron contraposiciones relacionadas con: (1) la mayor especialización de insumos del programa de maestría; (2) el papel del diseño e implementación de proyectos psicosociales en el pregrado; (3) la implementación diferencial de actividades de metacognición y reflexión sobre el proceso de aprendizaje; (4) el tipo y calidad de evidencia que los supervisores recaban para conocer el aprendizaje de sus supervisados, y (5) la necesidad de indicadores observables que den cuenta de las habilidades o competencias desarrolladas. Este modelo lógico es un antecedente apropiado para la formulación de preguntas investigativas que podrán responderse a futuro con un estudio de evaluación educativa.

Palabras clave: modelo lógico; supervisión; psicología clínica; concepciones.

\section{Abstract}

This qualitative study aimed to formulate a logic model describing the clinical supervision as an educational and programmatic activity. Data was collected by interviewing 10 clinical supervisors affiliated with a bachelor's program in psychology and a master's program in clinical psychology, offered by a privately-owned university located in Bogotá, Colombia. Answers were

* Hamer Bastidas-Bilbao y Ana María Velásquez, Centro de Investigación y Formación en Educación (CIFE), Universidad de los Andes, Bogotá, Colombia.

La correspondencia relacionada con este artículo debe ser dirigida a Hamer Bastidas-Bilbao, CIFE, Universidad de los Andes, carrera 1 \# 18A-12, piso 2, Bogotá, Colombia. Correo electrónico: hj.bastidas22@uniandes.edu.co

Cómo citar este artículo: Bastidas-Bilbao, H. \& Velásquez, A. M. (2016). Modelo lógico de la supervisión como actividad educativa para la formación en psicología clínica: concepciones de supervisores clínicos. Avances en Psicología Latinoamericana, 34(2), 293-314. doi: http://dx.doi.org/10.12804/ap134.2.2016.06 
analyzed following the NCT method (noticing things, collecting things, thinking about things). Descriptors of resources, activities and results were obtained in addition to the identification of these critical issues: (1) a higher specialization of resources used in the master's program; (2) the contribution of psychosocial projects at the bachelor's level; (3) a differential implementation of activities oriented towards development of metacognition and reflection on the learning process; (4) type and quality of evidence collected by supervisors in relation to the supervisees' learning outcomes, and (5) the need for designing and implementing observable indicators of skill and competence development. This logic model is an appropriate antecedent to the formulation of research questions that could be answered by conducting a further evaluation study of this program.

Keywords: logic model; supervision; clinical psychology; conceptions.

\section{Resumo}

Neste estudo qualitativo se derivou um modelo lógico da supervisão clínica como atividade educativa programática. Se efetuou uma entrevista semiestruturada a dez supervisores dos programas de graduação e mestrado de pós-graduação oferecidos por uma universidade privada em Bogotá, Colômbia. As respostas foram analisadas seguindo o método NCT (em inglês, noticing things, collecting things, thinking about things). Para além dos descritores de insumos, atividades e resultados, também se identificaram contraposições relacionadas com: (1) a maior especialização de insumos do programa de mestrado; (2) o papel da elaboração e implementação de projetos psicossociais na graduação; (3) a implementação diferencial de atividades de metacognição e reflexão sobre o processo de aprendizagem; (4) o tipo e qualidade de evidência que os supervisores arrecadam para conhecer a aprendizagem de seus supervisados; e (5) a necessidade de indicadores observáveis que deem conta das habilidades ou competências desenvolvidas. Este modelo lógico é um antecedente apropriado para a formulação de preguntas investigativas que poderão se responder no futuro com um estudo de avaliação educativa.
Palavras-chave: modelo lógico; supervisão; psicologia clínica; concepções.

En Colombia, las prácticas profesionales supervisadas constituyen una experiencia común a todos los programas de pregrado en psicología. Lo mismo ocurre en programas de posgrado con un énfasis profesionalizante. Más detalladamente, se han definido las prácticas profesionales, en su forma más concreta, como periodos de aprendizaje en los cuales los estudiantes ingresan a un entorno donde asumen labores profesionales (Baird, 2011; Wolfgang, 1976). Sin embargo, los estudiantes desarrollan sus actividades bajo la supervisión de un miembro de la planta docente o de un clínico con experiencia, ya que se parte del supuesto de que la supervisión de calidad enriquece el proceso de desarrollo profesional (Falender \& Shafranske, 2004; Morris \& Haas, 1984).

De hecho, en la literatura es posible encontrar una de las definiciones más exhaustivas de supervisión clínica, la cual fue propuesta por Falender y Shafranske (2004):

La supervisión es una actividad profesional particular en la cual la educación y el entrenamiento confluyen en el desarrollo de un ejercicio profesional informado científicamente y facilitado por un proceso interpersonal colaborativo. La supervisión implica observación, evaluación, retroalimentación, la facilitación de la autoevaluación por parte del supervisado, y la adquisición de conocimientos y habilidades por medio de la instrucción, el modelamiento y la resolución conjunta de problemas. Adicionalmente, la supervisión promueve la autoeficacia a través de una construcción sustentada en el reconocimiento de las fortalezas y talentos del supervisado. La supervisión asegura que la consulta clínica se ejecute de una manera competente en la que los estándares éticos, las prescripciones legales y las acciones profesionales se usen para promover y proteger el bienestar del 
consultante, la profesión, y la sociedad en conjunto.

(p. 3, traducción propia)

A partir de esta definición, es posible identificar cualidades fundamentales y componentes nucleares de la supervisión clínica. Dentro de estas cualidades fundamentales, se observa la confluencia de la educación y el entrenamiento, la cual evidencia que la supervisión clínica - incluso en los casos en los cuales se implementa en un contexto exclusivamente profesional - tiene una naturaleza colaborativa y educativa. En cuanto a sus componentes nucleares se identifican la observación, la evaluación, la retroalimentación y la autoevaluación como algunas de las actividades puestas en marcha durante un proceso de supervisión. Adicionalmente, la instrucción, el modelamiento y la resolución de problemas pueden ser acciones indicativas de una variedad de estrategias pedagógicas. Por último, el cumplimiento con las mejores prácticas y la protección del consultante, de la profesión y de la sociedad son indicadores de la naturaleza aplicada de la supervisión, así como de su vinculación a un contexto social y a la psicología clínica, como disciplina y profesión que se centra en el trabajo con individuos reales y sus asuntos particulares, percepciones y realidades.

De esta manera, comprender la supervisión y sus elementos evidencia el hecho de que el supervisor - a través de su quehacer - es responsable, por una parte, del bienestar de los consultantes que están atendiendo sus supervisados; y, por otra, de contribuir a un apropiado desarrollo de las competencias profesionales que se necesitan para ejercer la profesión de forma autónoma, responsable y efectiva (Malloy, Dobbins, Ducheny \& Winfrey, 2010) o para avanzar hacia programas de formación de posgrado. Sin embargo, en el contexto colombiano, el $60 \%$ de las personas que cuenta con un título profesional de pregrado en psicología no continúa con formación de posgrado alguna (Ocampo, Suárez, Fonseca \& Aguirre, 2012), debido parcialmente a que la legislación actual solo les exige a los profesionales la finalización de un pregrado (que dura entre cuatro y cinco años) antes de estar legalmente habilitados para ejercer en cualquier especialidad de la psicología. A pesar de esto, los programas de posgrado continúan emergiendo como una respuesta a una necesidad de un ejercicio profesional especializado y altamente competente, pero su cobertura y acceso son aún minoritarios.

En vista de esto, para la mayoría de quienes han completado un pregrado en psicología, las prácticas supervisadas son las únicas experiencias de entrenamiento que tienen en un contexto real, antes de ingresar al campo profesional como psicólogos completamente independientes y autónomos en su ejercicio. Así, la supervisión clínica - como componente central de las prácticas profesionales - constituye un cimiento estructural en el desarrollo profesional del psicólogo (Rusell \& Petrie, 1994; Association of State and Provincial Psychology Boards, 1998; Lizzio, Wilson \& Que, 2009). De hecho, la supervisión, en sí misma, funciona de forma equiparable a un programa - en este caso, un programa educativo- en el cual unos insumos se emplean de modo intencional para implementar ciertas actividades con el fin de alcanzar algunos resultados dentro de un contexto específico (McLaughlin \& Jordan, 2004), sea este de tipo social, disciplinar o profesional. Esto significa que la supervisión como actividad educativa debidamente estructurada es susceptible de ser evaluada, no solo por su importancia dentro de los procesos de formación de psicólogos, sino por la utilidad que un proceso sistemático de evaluación tendría a la hora de identificar la manera en la que la supervisión de prácticas clínicas contribuye al logro de los resultados educativos visionados por el oferente y los ejecutores del programa. Así mismo, habría de esperarse que un estudio evaluativo revelara las principales diferencias que la supervisión clínica tenga al avanzar desde un pregrado hacia un posgrado, con lo cual se haga aún más evidente la necesidad de facilitar el acceso a programas profesionalizantes de posgrado que se encuentren 
debidamente articulados en términos de sus insumos, actividades y resultados.

No obstante, este emprendimiento evaluativo no puede implementarse sin antes comprender, de forma profunda y sistemática, el funcionamiento de estos programas educativos estructurados como supervisión de prácticas clínicas. De hecho, en la literatura científica publicada en Colombia, y en Hispanoamérica en general, los referentes investigativos sobre el funcionamiento pedagógico o la contribución educativa de la supervisión clínica se encuentran en una escasez evidenciada en el número de publicaciones en bases de datos de los países hispanohablantes y Brasil. En particular, tras la revisión de la literatura regional se encontraron solo tres artículos en castellano (Castañeda-Cantillo, Abreo-Ortiz \& Parra-Benavides, 2006; Cruz-Fernández, 2009; Rodríguez \& Niño-Rojas, 2006). Dichas fuentes coinciden en abordar el problema de la formación de terapeutas desde una perspectiva sistémica, y aluden parcialmente a la supervisión, sobre todo para destacar el isomorfismo existente entre esta y los procesos psicoterapéuticos. En complemento, dentro de la literatura científica publicada en Brasil se hallaron seis ejemplares que abordaban directamente el estudio de la supervisión clínica en psicología para: (1) proponer modelos de supervisión análogos a marcos específicos de psicoterapia - psicodrama, psicoanálisis, humanismo, entre otros - (Teles-Tavora, 2002; Zaslavsky, Tiellet-Nunes \& Laks-Eizirik, 2003; Alvim-Saraiva \& Tiellet-Nunes, 2007; Boris, 2008); (2) brindar orientaciones para estructurar supervisiones centradas en casos clínicos específicos, como el abuso sexual en niños y adolescentes (Fortunato-Costa, Ribeiro, Penso \& Campos de Almeida, 2008), o (3) describir la implementación de supervisiones clínicas en instituciones públicas a la luz de las normativas legales vigentes (Mafacioli da Silva, Colomé-Beck, Costa de Figueiredo \& Cassol-Prestes, 2012).

En contraste, el estudio de la supervisión clínica como parte del proceso educativo de los psi- cólogos se ha realizado de forma más profusa en países anglosajones y europeos, con énfasis en la supervisión de prácticas profesionales — o internados - mayoritariamente en programas de doctorado. Este tipo de experiencias investigativas, si bien puede brindar luz en términos del funcionamiento de la supervisión dentro del marco disciplinar de la psicología clínica, no necesariamente ayuda a comprender la supervisión clínica, tal y como es implementada en Latinoamérica. Esto se debe a que, a diferencia de lo que ocurre en Estados Unidos, Canadá y Europa, los programas de pregrado de nuestras regiones habilitan plenamente a los portadores de sus diplomas para el ejercicio profesional autónomo e independiente. En este sentido, la formación de posgrado se convierte más en una decisión de formación voluntaria, antes que en un requerimiento legal para el ejercicio de la profesión. Esto hace aún más necesario estudiar las contribuciones que la supervisión clínica de prácticas profesionales tiene a la hora de satisfacer las necesidades educativas de los estudiantes que se forman como psicólogos.

Ahora, tras haber considerado algunas particularidades de la formación profesional en psicología en Latinoamérica, es preciso retornar al hecho de que la literatura científica producida en Hispanoamérica y Brasil tampoco conceptualiza la supervisión clínica en sí misma como un programa educativo conformado por insumos, actividades y resultados, a pesar de su evidente naturaleza educativa y, más aún, a pesar de su implementación casi exclusiva en los ámbitos formal, institucional y profesional, en el marco de la educación superior. La omisión de esta conceptualización programática podría explicar, a su vez, la ausencia en la literatura regional de experiencias que hayan evaluado sistemáticamente los aportes que la supervisión de prácticas clínicas puede tener a favor del logro de los resultados educativos visionados por sus oferentes y ejecutores. Esto se debe a que una implementación juiciosa de una evaluación educativa implica, sobre todo, reconocer la naturaleza programática de las 
actividades que se busca evaluar y, desde esa base, comprender de qué manera el programa de interés opera y cómo esas operaciones resultan - $\mathrm{O}$ noen unos logros concretos (McDavid \& Hawthorn, 2006). Más aún, el funcionamiento de un programa no transcurre de manera cerrada o aislada, ya que los programas educativos están enmarcados en un contexto sociohistórico y las interacciones con dicho contexto hacen que el programa se comporte como un sistema abierto (McDavid \& Hawthorn, 2006) y permanentemente susceptible de nuevas acomodaciones, cuya dinámica se evidencia y comprende a través de su evaluación.

Una forma útil de organizar anticipadamente el diseño de un estudio evaluativo es construyendo un modelo lógico del programa que se busca evaluar (McLaughlin \& Jordan, 2004). Más específicamente, un modelo lógico es una representación - gráfica o narrativa - que brinda información acerca de cómo un programa funciona en un momento dado del tiempo (McLaughlin \& Jordan, 2004; McDavid \& Hawthorn, 2006), es decir, cómo el programa incorpora unos insumos a partir de los cuales se estructuran unas actividades que, a su vez, producen unos resultados. Adicionalmente, la elaboración de un modelo lógico de forma antecedente al diseño e implementación de un estudio evaluativo es descrita como una parte importante dentro de cualquier proceso juicioso de evaluación de programas (McDavid \& Hawthorn, 2006). Esto se debe, en especial, a que la construcción de un modelo lógico también permite identificar el tipo de preguntas evaluativas que podrán ser abordadas, así como los elementos y evidencias que contribuirán a la obtención de respuestas metodológicamente válidas (McLaughlin \& Jordan, 2004). Más aún, un modelo lógico también contribuye a evidenciar y describir los supuestos subyacentes al funcionamiento del programa que se pretende evaluar, los cuales en su conjunto constituyen la teoría del programa (McLaughlin \& Jordan, 2004).

De esta manera, este estudio recorre un primer camino para establecer una nueva línea de investigación en la que confluyen la psicología, la educación y la evaluación de programas como disciplina. En consecuencia, se reporta una investigación que busca identificar cómo, mediante la elaboración de un modelo lógico de un programa de supervisión de prácticas clínicas, se evidencia un funcionamiento particular del programa y se identifican focos de evaluación educativa de carácter pedagógico que puedan tener repercusiones en la manera en la que se implementa el programa y en la manera en la que este contribuye al logro de los resultados educativos visionados por su oferente y sus ejecutores.

\section{Método}

Para la construcción del modelo lógico que aquí se reporta se emplearon metodologías de carácter cualitativo, debido al interés del investigador principal por acercarse a la realidad de los programas seleccionados — no solo como intérprete-, sino además como interlocutor de los actores que hacen parte integral de ellos (Stake, 2010). Además, dado que este estudio hace parte de una investigación general en evaluación educativa, se espera que el modelo lógico que se construya a partir de las concepciones de los supervisores participantes dé cuenta de focos evaluativos importantes. Por esto, a lo largo del análisis, y en reconocimiento de las limitaciones inherentes al uso de concepciones como fuente inicial de información, se hará hincapié en la identificación de aspectos que requerirán una observación directa de su ejecución, lo cual será realizado en otras fases de la investigación a fin de establecer una triangulación metodológica con la cual efectuar aseveraciones válidas respecto al funcionamiento del programa que concierne a este estudio.

El programa y los participantes seleccionados corresponden a los componentes de supervisión de prácticas clínicas de los programas de maestría profesionalizante y pregrado ofrecidos por una universidad privada con acreditación institucional de alta 
calidad ubicada en Bogotá, Colombia. Para acceder al programa y a los supervisores vinculados a este, se contactó la Dirección General, la Dirección de Desarrollo Profesional y la Dirección de Posgrados del Departamento de Psicología de la universidad participante para obtener los correspondientes avales institucionales. Luego, durante la segunda mitad de 2013, se invitó por correo electrónico a todos los supervisores clínicos del programa de maestría (6), así como a todos los supervisores del área clínica del programa de pregrado (8). De ese total, 13 supervisores aceptaron participar en el estudio; mientras que uno no respondió a las solicitudes de contacto. De los 13 supervisores que brindaron su consentimiento para participar, 10 aceptaron agendar $y$ responder a las actividades de esta investigación; mientras que los 3 supervisores restantes no pudieron ser vinculados al estudio dentro del periodo disponible para la recolección de datos. La participación voluntaria, las consideraciones de este estudio y su reporte, así como la aceptación del registro en audio quedaron debidamente consignadas por escrito en documentos de consentimiento informado.

Una vez los participantes brindaron su consentimiento informado, ellos diligenciaron una hoja de datos básicos en la cual se consignaba información acerca de su nivel de estudios, sus años de experiencia en supervisión y su entrenamiento específico como supervisores. Luego, se efectuó una entrevista semiestructurada basada en una guía con preguntas abiertas que se agrupaban en categorías preliminares correspondientes a insumos, actividades de enseñanza y aprendizaje, y resultados. Estos últimos, a su vez, se subdividían en productos, logros e impacto. Estas categorías se escogieron como un organizador previo de la información que se iba a recolectar, ya que corresponden a algunas de las categorías más comúnmente descritas dentro de los marcos de referencia para el modelamiento de la lógica de un programa (McLaughlin \& Jordan, 2004; McDavid \& Hawthorn, 2006).
Luego de efectuarse las entrevistas, se transcribieron sus respectivas audiograbaciones y los documentos resultantes se incorporaron al software Atlas.ti versión 7 para facilitar el proceso de identificación de frecuencias de códigos, segmentos de texto, relaciones y repeticiones (Friese, 2012). Tras esto, se empleó el método analítico NCT descrito por Seidel (1998, citado por Friese, 2012), el cual hace referencia a tres procesos básicos: notar elementos, recolectarlos y pensar acerca de ellos (en inglés: noticing things, collecting things y thinking about things). Más en detalle, el primer paso consistió en una revisión minuciosa de todos los datos que reposaban en las transcripciones con el fin de notar - mayoritariamente de forma descriptiva(Friese, 2012) todos aquellos hallazgos o datos que se relacionaban de forma preliminar con los interrogantes de investigación. Posteriormente, la fase de recolección de elementos corresponde en gran medida a lo que otros autores denominan codificación (Auerbach \& Silverstein, 2003) o suma categórica (Stake, 1998) y, tal como su nombre lo sugiere, consistió en agrupar elementos conceptualmente similares $-\mathrm{o}$ que reflejaban patrones de información - de tal manera que fue posible identificar unidades temáticas con un mayor nivel de abstracción (Auerbach \& Silverstein, 2003; Friese, 2012). Luego se procedió a integrar los hallazgos y a encontrar en ellos la mejor manera de acoplamiento que permitiera dar respuesta a las preguntas de investigación (Friese, 2012) a través de la construcción de una narrativa.

A la par del método NCT, se emplearon estrategias de triangulación (Stake, 1998, 2006, 2010) por interlocutor y teoría. Al triangular en conjunto con el interlocutor - member checking, en inglés-(Stake, 2010) se buscaba confirmar que las citaciones y los registros elaborados acerca de sus testimonios sean precisos y sensibles; entre tanto, para triangular la teoría fue necesario identificar e incluir puntos de vista teóricos que complementan los hallazgos de esta exploración. 


\section{Participantes}

Después de haber efectuado la tabulación de los formatos de información básica suministrados por los participantes, fue posible caracterizarlos en función de su edad, formación profesional y experiencia como supervisores. Específicamente, la edad promedio de los participantes se ubicó en 45.7 años y su experiencia como supervisores ha sido de 9.5 años. En relación con su nivel de estudios, 2 participantes poseían título de doctorado, 7 tenían título de maestría y uno se encontraba cursando una maestría. Finalmente, al pedir información acerca de entrenamientos específicos para realizar supervisión, ya sea durante la formación posgraduada o como educación continuada, 7 supervisores manifestaron no haber contado con dichas experiencias y solo 3 de ellos declararon haber recibido entrenamiento en supervisión durante su doctorado (2 participantes) o durante su maestría (un participante). Ellos también reportan haber participado en cursos de entrenamiento en supervisión con expertos internacionales.

\section{Hallazgos y discusión}

Mediante la elaboración de un modelo lógico de un programa de supervisión de prácticas clínicas incorporado dentro del pregrado y la maestría profesionalizante ofrecidos por la universidad participante, se busca aproximarse a la comprensión del funcionamiento particular de dicho programa, con el fin de identificar focos de evaluación educativa de carácter pedagógico. Para esto, se procedió a analizar las respuestas proporcionadas por los supervisores participantes, estableciendo conjuntos de categorías de información cuyos hallazgos se muestran a continuación de forma narrativa. Además, para preservar la confidencialidad de la participación, se aludirá a todos los supervisores usando pronombres masculinos y sus identidades se han codificado con las letras A, B, C y D, en referencia a los supervisores del programa de maestría, y con los números $1,2,3,4,5$ y 6 para citar a los supervisores del programa de pregrado.

\section{Insumos}

El primer elemento del modelo lógico (McDavid \& Hawthorn, 2006; McLaughlin \& Jordan, 2004) que los supervisores conciben acerca de la supervisión corresponde a los insumos. Según McDavid y Hawthorn (2006), los insumos son los recursos requeridos para que un programa educativo pueda operar. En este estudio, los insumos serán entendidos como todo aquel elemento o condición que existe o está disponible en el entorno para que las actividades inherentes a la supervisión puedan ejecutarse. Estos insumos se agruparon en tres categorías: técnicos-tecnológicos, institucionales y otros insumos. Los primeros se refieren a todos los elementos de uso profesional o herramientas de apoyo; los insumos institucionales aluden fundamentalmente a infraestructura o servicios propios de la universidad que se requieren para albergar las actividades de los programas, y los últimos agrupan aquellos insumos que no correspondan a las agrupaciones previas. Los insumos identificados por los supervisores se presentan en la tabla 1.

Los supervisores del programa de maestría reportan insumos con elementos técnico-tecnológicos e institucionales más especializados, tal como lo son los equipos de audio y videograbación, el apuntador electrónico, los laboratorios de psicología y los consultorios con circuito cerrado de video en los cuales es posible implementar en un mismo momento actividades tanto de consulta clínica como de supervisión. En contraste, las supervisiones de pregrado se fundamentan principalmente en el uso de literatura disciplinar y de material audiovisual de interés general que pueda vincularse al tipo de problemáticas que los estudiantes atestiguan durante su práctica profesional. El grado de especialización de los insumos empleados se explica en razón del énfasis profesionalizante de la maestría (Universidad de los Andes, 2008) en 
Tabla 1

Insumos para la implementación de la supervisión clínica en pregrado y maestría

\begin{tabular}{|c|c|c|c|c|c|}
\hline \multicolumn{6}{|c|}{ Modelo lógico de la supervisión clínica: insumos } \\
\hline \multicolumn{2}{|c|}{ Técnicos-tecnológicos } & \multicolumn{2}{|c|}{ Institucionales } & \multicolumn{2}{|c|}{ Otros } \\
\hline Pregrado & Maestría & Pregrado & Maestría & Pregrado & Maestría \\
\hline $\begin{array}{l}\text { - Literatura disci- } \\
\text { plinar }(1,2,3,4 \text {, } \\
5,6) \\
\text { - Videos de entre- } \\
\text { namiento }(2) \\
\text { - Videobeam }(3) \\
\text { - Documentales } \\
(4,5)\end{array}$ & $\begin{array}{l}\text { - Literatura disciplinar } \\
\text { (A, D) } \\
\text { - Equipos de audio } \\
\text { y videograbación } \\
\text { (A, C, D) } \\
\text { - Apuntador (A, C, D) } \\
\text { - Protocolos de inter- } \\
\text { vención clínica (B, D) } \\
\text { - Guías de formula- } \\
\text { ción (B) } \\
\text { - Instrumentos de eva- } \\
\text { luación (D) }\end{array}$ & $\begin{array}{l}\text { - Cámara de } \\
\text { Gesell (2) } \\
\text { - Biblioteca } \\
(1,2,3,4 \text {, } \\
5,6)\end{array}$ & $\begin{array}{l}\text { - Cámara de Gesell } \\
\text { (A, D) } \\
\text { - Biblioteca (A, B, D) } \\
\text { - Consultorios con } \\
\text { circuito de video } \\
\text { (A, C) } \\
\text { - Simultaneidad de } \\
\text { horarios de consul- } \\
\text { ta y supervisión (C) } \\
\text { - Laboratorios (D) }\end{array}$ & $\begin{array}{l}\text { - Películas de } \\
\text { interés general } \\
(1,4,6) \\
\text { - Profesionales } \\
\text { invitados (5) }\end{array}$ & $\begin{array}{l}\text { Relatos de la } \\
\text { experiencia } \\
\text { del supervisor } \\
\text { (A) }\end{array}$ \\
\hline
\end{tabular}

Nota: las letras A, B, C y D representan a los supervisores del programa de maestría y los números 1, 2, 3, 4, 5 y 6 hacen referencia a los supervisores del programa de pregrado.

contraste con el énfasis disciplinar del programa de pregrado (Universidad de los Andes, 2014). En consecuencia, el tipo de insumos reconocidos por los supervisores es congruente con el propósito de formación (general o especializada) que es característico de cada programa.

\section{Actividades}

Las actividades corresponden a todas las acciones efectuadas por el programa. A su vez, es posible agruparlas en componentes de acuerdo con su función y, a través de ellas, en conjunto, se busca dar lugar a unos resultados (McDavid \& Hawthorn, 2006). A efectos de esta investigación se proponen los siguientes: componente de comprensión representacional (Perkins, 1998), es decir, todas aquellas actividades en las cuales el propósito central yace en la identificación y comprensión de los conocimientos psicológicos; componente de comprensión funcional (Perkins, 1998), incluye actividades en las que el propósito fundamental corresponde a la aplicación contextualizada de los conocimientos de la psicología como disciplina, y componente de metacognición y reflexión (Moon,
2004), el cual abarca las actividades con las que se busca desarrollar una capacidad autovalorativa orientada al reconocimiento del propio proceso de aprendizaje y su impacto individual. También se identificaron categorías emergentes que resultaron útiles para otras agrupaciones de actividades: componente de actividades de monitoreo, evaluación y retroalimentación, que están diseñadas para moldear el desempeño contextualizado del supervisado de acuerdo con unos estándares profesionales, necesidades o propósitos educativos; por último, componente de organización de la experiencia de aprendizaje, que incluye actividades hechas para establecer una secuencia, ruta o estructura de las acciones que se emprenderán durante la supervisión.

Habiendo identificado los insumos empleados en las supervisiones, es posible explorar las actividades que los supervisores identifican dentro del modelo lógico de la supervisión clínica (tabla 2).

El análisis de los componentes de actividades implementadas por los supervisores participantes refleja puntos de concordancia y contraposición que dan información sobre la naturaleza de cada programa y el tipo de acciones pedagógicas implementadas para cada uno de ellos. En cuanto a 
Tabla 2

Actividades de implementación de la supervisión clínica en pregrado y maestría

\begin{tabular}{|c|c|c|}
\hline \multicolumn{3}{|c|}{ Modelo lógico de la supervisión clínica: actividades } \\
\hline Componentes & Pregrado & Maestría \\
\hline $\begin{array}{l}\text { Comprensión } \\
\text { representacional }\end{array}$ & $\begin{array}{l}\text { - Discusión grupal de temas disciplinares }(1,2,3 \text {, } \\
4,5,6) \\
\text { - Revisión de protocolos de evaluación (6) } \\
\text { - Análisis documentales disciplinares (5) }\end{array}$ & $\begin{array}{l}\text { - Discusión grupal de temas disciplinares (A, B, C, } \\
\text { D) } \\
\text { - Revisión de protocolos clínicos (B, C, D) }\end{array}$ \\
\hline $\begin{array}{l}\text { Comprensión } \\
\text { funcional }\end{array}$ & $\begin{array}{l}\text { - Modelamiento }(1,5) \\
\text { - Juego de roles }(1,2,5,6) \\
\text { - Presentación de caso clínico }(3,4,6) \\
\text { - Genograma }(1) \\
\text { - Diseño e implementación de proyecto psicoso- } \\
\text { cial o de revisión }(1,4,5,6)\end{array}$ & $\begin{array}{l}\text { - Modelamiento (A, B, C, D) } \\
\text { - Juego de roles (A, B, C, D) } \\
\text { - Formulación clínica (B, C) } \\
\text { - Presentación de caso clínico (C) }\end{array}$ \\
\hline $\begin{array}{l}\text { Metacognición y } \\
\text { reflexión }\end{array}$ & $\begin{array}{l}\text { - Diálogo socrático (5) } \\
\text { - Discusión de situaciones con impacto personal } \\
(2,3,4,6) \\
\text { - Análisis de películas relevantes a la práctica pro- } \\
\text { fesional (3) } \\
\text { - Discusión de situaciones de conflicto profesional } \\
(2,4,5,6) \\
\text { - Discusión del proceso de adaptación al entorno } \\
\text { de práctica }(1,3,4,5,6)\end{array}$ & $\begin{array}{l}\text { - Diálogo socrático (D) } \\
\text { - Discusión de situaciones con impacto personal } \\
\text { (A) } \\
\text { - Discusión de experiencias propias del supervisor } \\
\text { (A) } \\
\text { - Discusión de situaciones de conflicto profesional } \\
\text { (A) } \\
\text { - Discusión de aprendizajes de la sesión (D) }\end{array}$ \\
\hline $\begin{array}{l}\text { Monitoreo, } \\
\text { evaluación y } \\
\text { retroalimentación }\end{array}$ & $\begin{array}{l}\text { - Discusión de reportes verbales de casos clínicos } \\
\text { del supervisado }(1,2,3) \\
\text { - Planeación de acciones clínicas }(2,3,4,5) \\
\text { - Comunicación de valoraciones del supervisor } \\
\text { sobre el desempeño del supervisado }(1,4,5,6) \\
\text { - Solicitud de retroalimentación sobre la supervi- } \\
\text { sión y el supervisor }(1) \\
\text { - Comunicación de valoraciones del supervisor y } \\
\text { los pares sobre el desempeño del supervisado en } \\
\text { tareas específicas }(2,3,5,6) \\
\text { - Revisión de documentos de un caso clínico (6) }\end{array}$ & $\begin{array}{l}\text { - Observación directa del supervisado y retroali- } \\
\text { mentación sucesiva }(\mathrm{A}, \mathrm{B}, \mathrm{C}) \\
\text { - Observación y retroalimentación de videos del } \\
\text { supervisado (A, D) } \\
\text { - Discusión de reportes verbales de casos clínicos } \\
\text { del supervisado (A, B, C) } \\
\text { - Planeación de acciones clínicas (A, B, C) } \\
\text { - Comunicación de valoraciones del supervisor } \\
\text { sobre el desempeño del supervisado (A, D) } \\
\text { - Solicitud de retroalimentación sobre la supervi- } \\
\text { sión y el supervisor (B) } \\
\text { - Observación y retroalimentación simultánea con } \\
\text { apuntador (C, D) } \\
\text { - Revisión de documentos de un caso clínico (D) }\end{array}$ \\
\hline $\begin{array}{l}\text { Organización de } \\
\text { la experiencia }\end{array}$ & $\begin{array}{l}\text { - Establecimiento y verificación de compromisos } \\
\text { de aprendizaje }(1,3,4,5,6) \\
\text { - Construcción de objetivos de aprendizaje }(3,5,6) \\
\text { - Construcción de plan de trabajo }(3,5,6)\end{array}$ & $\begin{array}{l}\text { - Establecimiento y verificación de compromisos } \\
\text { de aprendizaje (B) } \\
\text { - Agenda de sesión (A, B, D) } \\
\text { - Identificación de situaciones prioritarias (B) } \\
\text { - Construcción de objetivos de aprendizaje (B, C, D) }\end{array}$ \\
\hline
\end{tabular}

Nota: las letras A, B, C y D representan a los supervisores del programa de maestría y los números 1, 2, 3, 4, 5 y 6 hacen referencia a los supervisores del programa de pregrado.

las actividades centradas en la comprensión representacional, se observa una congruencia en la implementación de discusiones grupales acerca de temas propios de la disciplina, lo cual significa que incluso en el programa de maestría - que tienen un énfasis profesionalizante- el espacio de supervisión también se constituye como un ambiente de aprendizaje donde es posible identificar 
y comprender los conocimientos psicológicos que sustentan las habilidades y competencias que deben desarrollar los supervisados. En contraste, a manera de contraposición entre los dos programas, solo los supervisores de maestría mencionan efectuar revisiones de protocolos de intervención clínica, lo que da cuenta de un proceso de aprendizaje representacional que bien puede ser precursor del desarrollo de una comprensión funcional directamente ligada a las acciones clínicas que los estudiantes desarrollarán en contextos auténticos de desempeño y que, como ya se ha mencionado, es compatible con la naturaleza de profundización del programa de maestría.

Por otra parte, en relación con las actividades centradas en la comprensión funcional, todos los supervisores de maestría describen implementar actividades de modelamiento y juego de roles en las cuales se requiere la acción del supervisado con el fin de evidenciar su grado de desarrollo de habilidades - ya sean de entrevista, evaluación, formulación o intervención-. Estas actividades también se implementan en alguna medida en las supervisiones de pregrado. No obstante, eventualmente en las supervisiones de pregrado se toma un rumbo distinto para llegar a focalizarse en el diseño e implementación de un proyecto psicosocial diseñado e implementado por los supervisados. Este tipo de proyectos surge del interés del supervisado y deben responder a una necesidad identificada por ellos en sus respectivos contextos de práctica, a la vez que rescatan o dan continuidad a los trabajos realizados por supervisados anteriores (supervisores 1, 4, 5 y 6). Uno de los supervisores clarifica la naturaleza de estos proyectos:

[...] las primeras veces que tenemos supervisión hablamos sobre cómo ellos van viendo [...] el contexto [y] lo que ellos van viendo que necesitan [los usuarios]. Ya después ellos llevan la propuesta [para su proyecto] [...] y entonces esa la discutimos entre todos y ya ahí se empieza [...]. Los criterios en los cuales yo apruebo los proyectos es que tengan una pertinencia teórica y una pertinencia social. Es decir, "si esa investigación teórica que tú vas a hacer le va a servir al siguiente grupo de practicantes que venga para poner en práctica algo, perfecto. Pero si solamente se va a quedar en ti y no lo vas a compartir con nadie, ¿para qué lo haces?, pues a ti te puede interesar mucho, pero no puedes funcionar de forma aislada a tu contexto de práctica" (Supervisor 1).

Adicionalmente, al analizar el componente de metacognición y reflexión, se identifica otra divergencia entre las supervisiones de los programas de pregrado y maestría, expresada en la multiplicidad de actividades que los supervisores de pregrado reportan implementar para el abordaje de todos los asuntos relacionados con el impacto personal, las situaciones de conflicto profesional y el proceso de adaptación que los supervisados viven en función de los acontecimientos de su práctica profesional. Este tipo de actividades son reportadas en menor medida por los supervisores de maestría, y ello pone en evidencia que la supervisión de pregrado también parece cumplir una función reiterada de acompañamiento de los supervisados durante su proceso de transición desde la universidad hacia el contexto laboral; sin embargo, dicha función es descrita y conceptualizada en la literatura como mentoring y no es equivalente a la supervisión clínica, a pesar de la superposición de algunas de sus características (Milne, 2009). Ahora, en cuanto a las actividades del componente de metacognición, un supervisor de pregrado y uno de maestría mencionan implementar formas de diálogo socrático donde, a partir de una indagación guiada, el supervisado logra generar formas de razonamiento y planes de acción, de tal manera que estos no sean simplemente dictaminados por el supervisor. No obstante, a partir de los reportes de los supervisores entrevistados, no es posible identificar otras actividades que contribuyan al desarrollo de una metacognición de los supervisados acerca de sus propios aprendizajes. 
Por otra parte, en relación con las actividades de evaluación y retroalimentación, tanto los supervisores de pregrado como de maestría coinciden en la implementación de discusiones a partir de los reportes verbales que los supervisados hacen de sus casos clínicos. Igualmente, la supervisión incluye actividades en las cuales el supervisor da su valoración general sobre el desempeño del supervisado. No obstante, a manera de contraposición, los supervisores de maestría describen efectuar observaciones directas de las acciones clínicas del supervisado, ya sea en vivo o a través de videograbaciones. Incluso, algunos supervisores reportan complementar sus observaciones en vivo con retroalimentaciones simultáneas mediante el uso de un apuntador electrónico. Ninguna de estas actividades es reportada por los supervisores de pregrado, lo que se explica a partir del hecho de que ellos no están vinculados directamente con las instituciones donde sus supervisados realizan sus prácticas profesionales, lo cual dificulta, e incluso impide, que ellos accedan a una observación directa de las acciones clínicas de los estudiantes. La principal implicación de que el supervisor no pueda acceder a la observación directa del supervisado en su contexto corresponde a que no es posible evaluar el desempeño mediante evidencias directas y, en su lugar, deben emplearse evidencias indirectas (por ejemplo, autorreportes, reportes de terceros, etc.) que han sido descritas en la literatura como insuficientes para establecer la efectividad de la supervisión y el proceso de aprendizaje de los supervisados (Bernard \& Goodyear, 2004).

A pesar de esto, es interesante mencionar el énfasis que los supervisores de pregrado hacen a la hora de describir actividades de retroalimentación, donde uno de los supervisados recibe las valoraciones hechas no solo por su supervisor, sino por sus pares. Todo en relación con el desempeño exhibido durante una actividad específica (por ejemplo, presentación de un caso, orientación de una discusión, socialización de un proyecto psicosocial). Este tipo de actividades puede interpretarse como un indicador de una dinámica grupal formativa y colaborativa fomentada por acción directa del supervisor y de las actividades diseñadas por él. Al contrario, el papel del grupo de supervisados no emerge como un tema recurrente entre los supervisores del programa de maestría y tampoco es posible inferir que este tipo de actividades se esté implementando en sus supervisiones, a pesar de que el formato grupal de supervisión tiene impactos positivos en términos de mayor independencia del supervisor, mayor relevancia de las retroalimentaciones y apoyo emocional entre pares (Goodyear \& Nelson, 1997).

La última categoría de actividades del modelo lógico de la supervisión corresponde a aquellas focalizadas en la organización de la experiencia de aprendizaje. En esta agrupación, la principal congruencia entre los supervisores de pregrado y maestría está dada por la realización de actividades para la construcción de objetivos. Esto significa que el tipo de aprendizajes que está por desarrollar se construye colaborativamente y no viene detallado de antemano. Sin embargo, las supervisiones de pregrado ponen el relieve en la construcción de un plan de trabajo (sobre el cual se sustenta el proyecto psicosocial de los supervisados, así como otras actividades de la práctica profesional) y en efectuar una verificación de los compromisos de aprendizaje derivados de este. Tales actividades dan cuenta de un mayor grado de monitoreo y direccionamiento del supervisado, lo cual es congruente con un modelo desarrollista de la supervisión (Falender \& Shafranske, 2004; Milne, 2009), donde hay una serie de etapas caracterizadas por niveles progresivos de autonomía del supervisado e intervención del supervisor. El establecimiento y la verificación de los compromisos de aprendizaje no son temas emergentes entre los supervisores de maestría y, en su lugar, se identifica una actividad de estructuración de las sesiones de supervisión — por medio del establecimiento de una agenda de sesióncon la cual se focaliza el espacio de supervisión en unas actividades claramente definidas, lo que 
se reconoce como una actividad propia de un modelo cognitivo-conductual (Bernard \& Goodyear, 2004; Falender \& Milne, 2009; Shafranske, 2004; Watkins, 1997).

\section{Resultados}

Esta categoría abarca no solo productos, es decir, elementos cuya elaboración resulta de las actividades naturales de los programas (McDavid \& Hawthorn, 2006), sino efectos, los cuales se entienden como cambios o transformaciones (inmediatas y demoradas) que recaen en el entorno de los programas y que son atribuibles a la ejecución de sus actividades, las cuales son vivenciadas tanto por sus usuarios como por sus beneficiarios (McDavid \& Hawthorn, 2006).

Más detalladamente, los supervisores de los programas de pregrado y maestría reportan una serie de productos fundamentalmente de tipo documental, cuyo contenido permite diferenciarlos en documentos clínicos, documentos organizacionales y documentos para el paciente o el servicio de práctica profesional. Los documentos clínicos dan cuenta de las características de los casos abordados por los supervisados o de las acciones clínicas emprendidas por ellos. Un tipo de producto documental adicional corresponde a los documentos organizacionales, donde se reportan los aspectos formales de la práctica supervisada, que incluyen planeación y reporte de labores, bases de datos, entre otros. Por último, sumándose a los productos documentales, los programas de pregrado y maestría también resultan en productos dirigidos al paciente o al servicio psicológico al cual se encuentren vinculados los supervisados. Estos productos para el paciente o el servicio consisten en talleres, sesiones clínicas y documentos informativos. Todos los productos identificados por los supervisores se presentan de forma esquemática en la tabla 3.

Los productos descritos por los supervisores indican que en el programa de pregrado hay un mayor reconocimiento a la elaboración de productos documentales donde se planean las sesiones o las actividades clínicas que van a ser implementadas por los supervisados. En contraste, en el programa

Tabla 3

Productos de la supervisión clínica en pregrado y maestría

\begin{tabular}{|c|c|c|}
\hline \multicolumn{3}{|c|}{ Modelo lógico de la supervisión clínica: productos } \\
\hline Productos & Pregrado & Maestría \\
\hline $\begin{array}{l}\text { Documentos } \\
\text { clínicos }\end{array}$ & $\begin{array}{l}\text { - Plan de sesión o actividad clínica }(1,2,5,6) \\
\text { - Historias }(1,2,4) \\
\text { - Informe de caso }(1,4,6) \\
\text { - Cierre de caso }(1)\end{array}$ & $\begin{array}{l}\text { - Resumen semanal de caso (A) } \\
\text { - Agenda y plan de sesión (A) } \\
\text { - Guías de formulación (B, C, D) } \\
\text { - Historias (B, D) } \\
\text { - Informe de caso (B) } \\
\text { - Cierre de caso (B) } \\
\text { - Remisiones (D) } \\
\text { - Notas de sesión (D) }\end{array}$ \\
\hline $\begin{array}{l}\text { Documentos } \\
\text { organizacionales }\end{array}$ & $\begin{array}{l}\text { - Plan semestral de práctica }(2,3,5,6) \\
\text { - Informe semestral de práctica }(2,4,5,6) \\
\text { - Bases de datos }(6)\end{array}$ & $\begin{array}{l}\text { - Formato de cumplimiento de horas (A) } \\
\text { - Acta de sesión de supervisión (D) } \\
\text { - Contrato de supervisión (D) }\end{array}$ \\
\hline $\begin{array}{l}\text { Para el paciente } \\
\text { o el servicio }\end{array}$ & $\begin{array}{l}\text { - Talleres }(1,2,3,5,6) \\
\text { - Documentos informativos }(3,4,6) \\
\text { - Sesiones clínicas }(5) \\
\text { - Pilotaje de protocolos }(6)\end{array}$ & - Sesiones clínicas (C) \\
\hline
\end{tabular}

Nota: las letras A, B, C y D representan a los supervisores del programa de maestría y los números 1, 2, 3, 4, 5 y 6 hacen referencia a los supervisores del programa de pregrado. 
de maestría uno de los productos reconocido de forma recurrente y sistemática por los supervisores consiste en las guías de formulación clínica. A pesar de que el grado de especificidad es distinto, ambos productos dan cuenta de un proceso sistemático en el diseño de las actividades de relevancia clínica que serán administradas por los supervisados junto a sus consultantes.

En cuanto a los documentos organizacionales, estos emergen de forma recurrente y sistemática en los testimonios de los supervisores de pregrado. Específicamente, ellos hacen referencia a un plan semestral de actividades que se elabora durante el inicio del periodo de práctica supervisada y el cual, eventualmente, se convierte en un informe final. No obstante, la identificación de este tipo de documentos organizacionales no emerge con la misma frecuencia entre los supervisores de maestría, ya que uno de ellos menciona la existencia de un registro de cumplimiento de horas; mientras que otro describe la elaboración de un contrato de supervisión y de actas de sesión. Lo descrito por ellos no permite establecer si estos documentos dan cuenta de una práctica común al programa o si responden a aspectos particulares implementados individualmente por cada supervisor.

En relación con los productos para el paciente, los supervisores de pregrado describen un tema emergente y estable relacionado con la elaboración de talleres. Dichos talleres abordan distintas problemáticas o necesidades de los consultantes o de la institución prestadora de servicios psicológicos. Los talleres son ideados por los supervisados, cuentan con retroalimentación por parte del supervisor -en algunos casos incluso por parte de sus pares y jefes inmediatos - y se implementan a lo largo del periodo de práctica profesional. En el programa de pregrado también se elaboran documentos informativos - como guías, folletos, entre otros - que buscan comunicar al paciente, en un lenguaje relevante y comprensible, información con valor psicológico que sirva de respaldo al proceso clínico por el cual pasan los consultantes.
Ahora, en relación con el programa de maestría, es interesante notar cómo uno de los supervisores menciona que las sesiones clínicas son un producto de la supervisión. Al respecto, es claro que los supervisados implementan de modo permanente las sesiones clínicas; en este sentido, ellas podrían verse mejor como una actividad del programa. No obstante, el supervisor entrevistado hace una precisión y muestra cómo, en efecto, la supervisión puede resultar en un diseño específico de sesiones clínicas que cumpla con unos lineamientos y unos estándares (supervisor C). Esta visión particular puede interpretarse como un indicador que refleja la manera en la que las supervisiones transforman la acción clínica del supervisado.

Adicionalmente, las concepciones de los supervisores participantes son indicativas de que los productos que resultan tras la implementación de las actividades de supervisión en los programas de pregrado y maestría no son solo un repositorio de información, sino que, a su vez, son fuentes de evidencia de cómo los supervisados desarrollan sus aprendizajes a lo largo de la práctica supervisada. A su vez, dichos aprendizajes, plasmados en productos o en acciones clínicas, pueden evidenciarse - según las concepciones de los supervisores - a través de cambios cognoscitivos o conductuales. Entre los cambios cognoscitivos, los supervisores identifican aquellos relacionados con el manejo de la información a través de síntesis (supervisor A), planeación (supervisor A, 6), conceptualización (supervisor A, D, 1, 6), asimilación (supervisor 1, $2,6)$, así como a través del propio reconocimiento de necesidades de aprendizaje (supervisor 4). Más detalladamente, los entrevistados señalan:

Pues la primera evidencia es [que ellos] “están planeando", están registrando la información. No solo [ya] no la están dejando o improvisando para llegar a la supervisión, sino que eso me da cuenta de que ellos preparan la supervisión. [...] [Además], uno a través de la planeación que [ellos] hacen de un tratamiento también identifica el nivel 
de apropiación del conocimiento que hay y cómo [este] se va acrecentando [y] modificando en el tiempo (Supervisor A).

[Yo identifico evidencias del aprendizaje de los supervisados] pues en las descripciones que ellos hacen. Si tú comparas uno con otro [...], por ejemplo, en una hipótesis que [el supervisado] hizo al comienzo del semestre con una hipótesis explicativa que hacen durante el semestre [...], la hipótesis de comienzo de semestre es una hipótesis lineal, desde el sentido común. Por lo general contemplan pocos factores que influyen en la problemática, es una hipótesis que da pocas posibilidades de intervención mientras que la hipótesis de final de semestre contempla más factores de influencia del problema, visualiza posibilidades de acción, tiene componentes teóricos claros (Supervisor 1).

Digamos que [...] un[a] [evidencia es] que ellos mismos confiesan que hay cosas que no conocían. Ellos mismos dicen: "me volví un ocho pero aquí está, espero haberlo hecho bien" y como son [productos] cortitos entonces nos sentamos, los leemos, incluso a veces yo mismo se los devuelvo y ellos dicen: "ah sí, claro... [debo mejorar] redacción... Dios mío". [...] Como que ellos mismos lo confiesan: "uy, mira, me volví un ocho, yo no había visto esto" y nos sentamos y lo hablamos y [lo] vemos (Supervisor 5).

Complementariamente, los entrevistados mencionan que entre los cambios conductuales que ellos identifican para evidenciar el aprendizaje de los estudiantes se encuentran el seguimiento de instrucciones (supervisor A, 3, 4, 6) y la ejecución o elaboración de sus acciones o productos clínicos (supervisor 1, 3, 5, 6). Al respecto, los supervisores detallan:

[Yo identifico evidencias del aprendizaje de los supervisados al] [...] ver qué tanto incorporaron las sugerencias que dimos [en] la sesión de supervisión anterior. Por ejemplo, dijimos: "con este paciente es importante trabajar tal cosa... Búscate esto... Y vamos a trabajar implementando el protocolo de Fairburn para los trastornos de la alimentación”. Entonces, eso me permite hacer seguimiento: "pero habíamos dicho que trabajábamos en esto... ¿qué pasó aquí?... ¿por qué no se trabajó?" (Supervisor A).

En esos formatos se puede ver perfectamente - o en los talleres o en lo que sea- se puede perfectamente ver cómo ellos van articulando la teoría [y] la práctica, cómo van enriqueciendo sus comprensiones, cómo van complejizando sus acciones... (Supervisor 1).

Estos dos tipos de cambios indicativos de aprendizaje se contrastan entre sí, de tal manera que el supervisor puede valorar de modo más integral el proceso de aprendizaje de los supervisados en su respectivo contexto de acción profesional o en la ejecución de una tarea que requiere una competencia clínica compleja (la formulación de caso o la evaluación psicosocial, por mencionar algunos ejemplos). Esto lo explican los supervisores de la siguiente manera:

La formulación tiene una particularidad y es que se puede llenar de una manera [...] verdadera, pero [...] sin tener toda la información relevante del paciente para formular las hipótesis de mantenimiento y el tratamiento. Pero, puede estar bien llena. Entonces, en la medida en que haya esa correspondencia entre la información que ha dado el paciente, [la] que hemos visto en los videos, [la] que hemos escuchado en las sesiones de grabación, [que] está consignada allí. Y [eso] permite de manera lógica plantear las hipótesis de mantenimiento y hay una relación de esas hipótesis de mantenimiento con las sesiones de intervención [...] (Supervisor C).

Entonces, por ejemplo, [durante] la primera semana en evaluación psicosocial ¿qué busco yo? [...] que [el supervisado] conozca el protocolo, que sepa por qué se hace, que discutamos el protocolo, en qué momento se utiliza, cómo nació, [...] qué opina [el supervisado], qué debe haber [en el protocolo], qué 
observa, cómo se debe llevar a cabo, qué preguntas tiene, cómo preguntarías tú esto. [...] Inicialmente, ellos tienen un cuadernito donde van anotando $[\mathrm{y}$ hay que] enseñarles cómo ir soltándo[se] un poco, cómo armar su estructura, cómo ir dejando ese formato [...]. Y eso es idealmente lo que busca uno porque cualquiera que logre desarrollar esto puede dedicarse a cualquier área de la psicología. [...] Además, [hay que] ver el ritmo del estudiante porque hay estudiantes que esto me lo hacen en una semana y hay estudiantes que esto lo pueden llevar en un mes (Supervisor 6).

En estas situaciones descritas por los supervisores, se observa cómo los cambios cognoscitivos y conductuales que ellos identifican a lo largo del proceso de aprendizaje de sus supervisados no se valoran aisladamente. Por ejemplo, un cambio en la asimilación del conocimiento disciplinar evidenciado en un documento se contrasta con la forma de administración de un taller clínico — o elaboración de un producto - para pacientes. Dicho contraste puede, desde la perspectiva de los supervisores, hacer evidenciables las acciones del supervisado que son congruentes con los elementos disciplinares.

De esta manera, tras haber analizado cómo los productos hacen parte de la dimensión de resultados del modelo lógico de la supervisión, es posible identificar los efectos o transformaciones atribuibles a la implementación de los programas que son vivenciados por los usuarios y beneficiarios de estos. Específicamente, los usuarios de los programas son los supervisados, quienes se vinculan formalmente a los programas de pregrado o maestría ofrecidos por la universidad participante. En complemento, el conjunto de beneficiarios del programa es más extenso y abarca a los pacientes que reciben atención clínica, las instituciones de salud que albergan a los supervisados y otras personas que se relacionen directamente con ellos - incluso si estos son los mismos usuarios, o los profesionales a cargo de la implementación de los programas-. Sin embargo, los efectos del programa también pueden recaer en la psicología como disciplina y profesión, sin identificar personas en particular.

En cuanto a los resultados del programa experimentados por los supervisados — según las concepciones de los supervisores - se observa que estos abarcan aspectos directamente relacionados con el desarrollo de habilidades y competencias clínicas, la autoeficacia del supervisado y su capacidad de gestionar autónomamente su quehacer profesional de manera continuada y alineada con los estándares de la profesión. Respecto al desarrollo de habilidades y competencias clínicas, los supervisores de pregrado y maestría aluden a la utilización del conocimiento disciplinar (supervisor B, C, D, 1), la conceptualización del caso (supervisor A), la formulación (supervisor B, D), las habilidades básicas de comunicación verbal y no verbal (supervisor B, D, 1, 3) y la intervención terapéutica (supervisor A, B, D) para describir el tipo de aprendizajes atribuible a las acciones del programa. En relación con la autoeficacia del supervisado, los supervisores describen cómo la supervisión, a través del desarrollo de habilidades y competencias, también resulta en una mejor percepción que los supervisados tienen acerca de su solidez y capacidad de ofrecer servicios psicológicos (supervisor B, 2, 5), reforzada por una mayor tolerancia a la incertidumbre y percepción de control sobre su labor (supervisor D). En tercera instancia, en relación con la gestión autónoma del quehacer profesional, los supervisores de maestría van más allá de los aprendizajes de habilidades clínicas y la autoeficacia de los supervisados, y añaden que el paso por el programa les permite a ellos desarrollar un sentido de responsabilidad que conlleva el reconocimiento de la importancia del contacto y el apoyo profesional para el manejo de los casos (supervisor A, D), el cumplimiento de los estándares profesionales (supervisor C), así como de la autoevaluación (supervisor B, D) y la formación continuada (supervisor A). En cuanto a los distintos tipos de resultados ya mencionados, los supervisores relatan: 
Yo creo que lo que le ofrece la supervisión a los estudiantes es el sentido de responsabilidad acerca de su ejercicio profesional y de que la supervisión debería mantenerse incluso una vez terminado el proceso de formación de la maestría. [Esta es] la responsabilidad por ofrecer un servicio de calidad a un usuario en donde es importante mantener un contacto con un profesional, o con un pool de profesionales, con los cuales uno pueda discutir sus casos. [...] Otra de las ganancias que ofrece la supervisión es el enriquecimiento en la mirada del supervisado [acerca] del caso, distintas perspectivas para ver y manejar[lo] [...], [así como] un enriquecimiento también en sus habilidades obviamente, pero también en las estrategias de abordaje (Supervisor A).

[Los estudiantes desarrollan] ante todo una actitud proactiva y de búsqueda de información, eso es una característica que quiero que aprendan. En segundo lugar, aprenden habilidades específicas: cómo se estructura, qué se dice, etc. En tercer lugar, aprenden a evaluar, a autoevaluarse, a evaluar cómo está su ejecución, aprenden a ser autocorrectivos. En cuarto lugar, aprenden a desarrollar confianza en ellos, predictibilidad, ok, ese es el cuarto punto que espero que ellos aprendan. En quinto lugar, aprenden a interactuar con el supervisor y con otros compañeros de una manera profesional en torno a un caso particular (Supervisor D).

$\mathrm{Si}$ [tras el paso por la supervisión] el estudiante sale todavía más dubitativo, con menos confianza en sus capacidades pues no le fue muy útil la supervisión. Si el estudiante sale confiado, pues confiado y consciente también de sus limitaciones y de todo el camino que le toca por recorrer, pero [al mismo tiempo] claro en cuáles son sus fortalezas, sus debilidades, confiando desde la modalidad en la que yo me muevo, en el ser humano, y en los recursos del ser humano pues, [entonces], probablemente se ha contribuido a su formación de una manera eficaz (Supervisor 2).
En cuanto a los resultados de la supervisión clínica vivenciados por los beneficiarios de los programas, los supervisores de pregrado y maestría perciben que, en primer lugar, los consultantes se benefician de las prácticas clínicas supervisadas, ya que posibilitan su acceso a una atención de mayor calidad, planeada, asesorada por expertos y efectiva (supervisor A, B, C, 3) que responda a sus necesidades de atención psicológica (supervisor 1, $2,5,6)$. En sus palabras, los entrevistados mencionan: "[Los consultantes son beneficiarios porque] estamos garantizando, por un lado, que la atención que reciban haya sido digamos discutida por un equipo de expertos, que haya sido planificada... No la intervención espontánea que se le ocurrió al supervisado [...]" (supervisor A).

Cuando yo empecé a trabajar [... tenía un interés puntual en ese momento que era mi trabajo con [esta unidad médica] y curiosamente me llamaron para supervisar. [...] Yo quería que hubiera psicólogos para los pacientes con [esta problemática clínica]. Y hoy en día tengo como tres personas que yo he formado que son psicólogos de unidades [para el manejo de esta problemática clínica] y que han abierto la puerta. [...] Hoy en día tengo la satisfacción de saber que lo hacen y que lo hacen bien, en pro del paciente [...] (Supervisor 6).

En segundo lugar, los supervisores también se reconocen como beneficiarios de la implementación de las prácticas clínicas supervisadas. Para los supervisores de pregrado, este tipo de programas educativos les permiten mantener actualizados los conocimientos sobre la disciplina (supervisor $3,4,5,6)$. Adicionalmente, los supervisores de maestría dan cuenta de otros beneficios y mencionan que la implementación de los programas también trae como resultado el hecho de que ellos llegan a diversificar y desarrollar sus estrategias como supervisores (supervisor A, C), delimitar los objetivos que buscan alcanzar (supervisor B, C), 
recibir retroalimentación (supervisor $\mathrm{C}$ ) y ampliar su perspectiva dando cabida al punto de vista de los supervisados (supervisor D). Acerca de esto, los supervisores entrevistados relatan:

[Entre los beneficios que percibo de la supervisión considero que] cada vez pulo más el estilo de supervisión y las estrategias. Primero, limito más los objetivos, entonces no pretendo abarcar más de lo que creo que en ese tiempo se puede abarcar y eso a medida que pasa el tiempo es más fácil hacer. Y, el estilo, [es decir], eliminar cosas que no son las más efectivas y quedarme con las estrategias que voy viendo [que] son más efectivas. [Además], [...] el ejercicio mismo que hago [...] retroalimenta mi ejercicio del próximo semestre [...]. [También] me toca trabajar en lo que hay, logrando unos objetivos y [...] construyendo unos propósitos cada semestre distintos para hacer mejor mi trabajo, para ir creciendo en el trabajo que hago y pues desarrollándome como supervisor que es una labor y un ejercicio que he hecho durante toda mi vida profesional (Supervisor C).

Yo me beneficio mucho porque a mí me encanta el tema y yo digo que no sabría ni habría leído tanto ni tendría tanto material si no estuviera supervisándolos y eso me beneficia a nivel profesional, a nivel macro, porque estoy todo el tiempo actualizado. Me encanta leer artículos, todos los artículos que me traen ellos [yo les digo] “dénmelos, dénmelos, a mí me sirven" [y así] para la próxima [oportunidad] tengo banco de literatura. [Además, me beneficio al] compartir con otros profesionales y estar actualizado. [Por eso], creo que el beneficio es mutuo. Yo digo que si no hubiera un gran beneficio para mí yo no estaría supervisando de una forma como tan comprometida y no sería para mí tan satisfactorio hacerlo. Si yo fuera simplemente a hablar me cansaría, o sea, yo siento que cada vez igual recibo y que por eso crezco como supervisor (Supervisor 6).

En vista de lo anterior, el modelo lógico de la supervisión tal como es concebido por los super- visores también ilustra efectos atribuibles a su implementación que no recaen sobre individuos - sean estos los usuarios, los beneficiarios o los ejecutores-, sino sobre las instituciones, la comunidad en general, la disciplina y la profesión. Así, las instituciones educativas y de prestación de servicios psicológicos se benefician al contar con egresados o profesionales con un buen desempeño, lo cual redunda en su imagen favorable ante la sociedad (supervisor B, 2, 5, 6) y, adicionalmente, dichas instituciones también logran estructurar mejor los programas ofrecidos a sus usuarios primarios (supervisor C, D, 3, 4, 5, 6). En complemento, en el ámbito comunitario, las personas logran acceder a mejores servicios de psicología que desembocan en cambios individuales los cuales, a su vez, facilitan un mejor ajuste psicológico de las personas en sus entornos inmediatos (supervisor B, 2). En los planos disciplinar y profesional, los supervisores de los programas de pregrado y maestría consideran que la implementación de este tipo de programas de práctica clínica supervisada contribuye a la cualificación de los servicios de psicología clínica (supervisor A, B, C, 1, 4, 6), a la ampliación del campo profesional (supervisor $4,5,6)$ y a la sistematización y desarrollo de conocimiento psicológico junto a las mejores prácticas empleables para su difusión educativa (supervisor A, C). Para finalizar, con respecto a este conjunto de beneficios, y sus receptores, los supervisores comentan:

La disciplina [se beneficia] porque nosotros podemos formalizar, describir [...], [hacer] una formalización de la experiencia de la supervisión [para] que esto pueda volverse un ejercicio replicable, por ejemplo. Entonces, eso favorece el desarrollo de la disciplina y la generación de conocimiento sobre el quehacer del supervisor. Y, en el ámbito profesional, pues evidentemente se favorece la formación de personal más idóneo y en ese sentido se cualifica el ejercicio profesional de la psicología en el país, con miras a algo que se dé en algún momento y es 
una mayor regulación del ejercicio de la práctica clínica (Supervisor A).

En últimas yo creo que se beneficia la comunidad en general [...]. Me parece que es fundamental tener una muy buena supervisión porque eso podría favorecer que salgan profesionales mejor formados, entonces, si salen profesionales mejor formados pues van a ser mejores agentes de cambio. [También se beneficia] la comunidad en general porque pues si la comunidad en general puede acceder a mejores servicios de psicología clínica, una persona que acceda a mejores servicios puede tener mejores resultados consigo mismo. Y una persona que está mejor consigo mismo pues tiene un mejor impacto sobre el ambiente (Supervisor B).

Normalmente los estudiantes acaban dando cuenta de casos bellísimos [y] en las instituciones acaban fascinados con las cosas. [...] Las instituciones se agradan mucho [de] que les lleguen estudiantes, muchachos guiados de la manera en la que se hace en las supervisiones y yo diría que [...] eso era como muy unánime, como que gustaba mucho lo que pasaba con el muchacho gracias a una supervisión cuidadosa que se hace en psicología... que tú sabes que no se hace en otras carreras [...]. [Ellos] han ido transformando a los padres de familia, han logrado que los padres de familia sean más responsables, visiten más a los hijos, [todo] según lo que se esté buscando en cada taller (Supervisor 2).

\section{Conclusiones}

Este estudio se centró en la derivación de un modelo lógico basado en las concepciones que los supervisores clínicos de los programas de pregrado y maestría de la universidad participante tienen acerca del funcionamiento programático de la supervisión como actividad educativa para la formación de psicólogos clínicos. Como ya se ha mencionado, la construcción de un modelo lógico permite representar un programa educativo como un sistema abierto que está sustentado en unos insumos, a partir de los cuales se implementan unas actividades que dan cuenta de unos resultados en términos de productos, así como de efectos inmediatos y a largo plazo (McDavid \& Hawthorn, 2006; McLaughlin \& Jordan, 2004). El principal aporte de la construcción de un modelo lógico yace en la posibilidad de comprender a profundidad un programa de manera que se ilustre su funcionamiento interno, a fin de que los resultados se comprendan a la luz de las actividades implementadas y de los insumos con los que se cuente. En este caso, la construcción de un modelo lógico es un antecedente apropiado para el diseño y ejecución de una evaluación educativa (McDavid \& Hawthorn, 2006) que permita comprender a profundidad cómo la supervisión de prácticas clínicas — vista como un programa en sí mismo - contribuye al logro de los resultados educativos visionados por su oferente y sus ejecutores.

El modelo lógico basado en las concepciones identificadas en los supervisores participantes revela temas contrapuestos en relación con los insumos, las actividades y los efectos. En algunos casos, estas contraposiciones son el principal indicador para establecer focos de evaluación educativa para estudios posteriores, cuyos resultados se constituirán como una triangulación metodológica de las aseveraciones aquí presentadas. En términos de insumos, se identifica una utilización de insumos técnicos-tecnológicos e institucionales de mayor especialización en el programa de maestría en contraste con el programa de pregrado, lo cual es esperable debido a que el programa de pregrado tiene un énfasis disciplinar, mientras que la maestría posee un énfasis de profundización profesional (Universidad de los Andes, 2008, 2014).

Adicionalmente, al analizar los componentes de actividades de cada programa, se observa que el componente de comprensión funcional está organizado - en el caso del pregrado - en torno a un proyecto psicosocial diseñado e implementado por los supervisados de acuerdo con las necesidades identificadas en su contexto de práctica. Dicha organización en torno a un proyecto no es descrita por 
los supervisores de maestría. En consecuencia, un primer foco evaluativo consiste en la observación directa de las actividades estructuradas alrededor de los proyectos psicosociales de los estudiantes y los resultados que se derivan de estas, con el fin de establecer sus contribuciones diferenciales al proceso de aprendizaje de los supervisados.

Por otra parte, trayendo a consideración el componente de actividades centradas en la metacognición, debe mencionarse que la necesidad evaluativa fundamental de este componente consiste en su caracterización detallada ya que, a partir de las concepciones de los supervisores, no es posible evidenciar un tema recurrente que describa cuáles son las actividades que se implementan dentro de las supervisiones de pregrado y maestría para que el supervisado llegue a desarrollar una capacidad autovalorativa orientada al reconocimiento de su propio proceso de aprendizaje. En cuanto al componente de reflexión, los supervisores de pregrado mencionan de forma sistemática una multiplicidad de actividades orientadas a que el supervisado identifique el impacto individual que él experimenta a raíz de su proceso de aprendizaje y de su ejercicio profesional. No obstante, actividades de similar naturaleza no emergen sistemáticamente en las concepciones de los supervisores de maestría. En consecuencia, el foco de evaluación educativa para desarrollar en estudios posteriores consiste en la caracterización de las actividades orientadas a la reflexión que se estén implementando en las supervisiones del programa de maestría, su contraste con aquellas implementadas en el pregrado y la identificación de cómo dichos componentes de actividades contribuyen diferencialmente, o no, al proceso de aprendizaje de los supervisados de ambos programas de acuerdo con sus necesidades educativas particulares.

Adicionalmente, al analizar las contraposiciones identificadas en el componente de evaluación y retroalimentación, se identifica que los supervisores de maestría afirman recabar evidencias del proceso de aprendizaje de los supervisados a través de métodos directos (observación en vivo, observación de videograbaciones). Dicho proceso no lo describen los supervisores de pregrado, lo cual se explica parcialmente por el hecho de que los supervisores de este programa no necesariamente cuentan con acceso directo y permanente al campo de práctica profesional donde se encuentran los supervisados. De esta manera, una necesidad importante de evaluación educativa consiste en identificar el tipo y la pertinencia de las evidencias de aprendizaje que los supervisores de pregrado recaban de sus supervisados y cómo dichas evidencias permiten implementar actividades de retroalimentación formativa.

En tercera instancia, tras haber abarcado los insumos y las actividades del modelo lógico de la supervisión, es necesario describir las necesidades evaluativas que surgen a partir de las particularidades encontradas en la dimensión de resultados. Más detalladamente, al considerar los productos documentales y los productos para el paciente/servicio que los supervisores de pregrado y maestría describen de forma recurrente, surge una necesidad evaluativa de triangular con métodos observacionales el uso que se da a estos productos como parte de la supervisión y cómo estos se contrastan con cambios cognoscitivos y conductuales en el supervisado que permitan dar cuenta de su proceso de aprendizaje, su grado de desarrollo de habilidades y competencias y las acciones formativas que el supervisor implementa como respuesta a dichos cambios.

Para finalizar con la identificación de necesidades evaluativas en cada una de las dimensiones del modelo lógico de la supervisión es necesario construir e implementar estrategias de observación directa que permitan triangular los cambios que los supervisores conciben que experimentan los supervisados tras su paso por la práctica clínica supervisada. Con esto será posible evidenciar la manera en la que los supervisados desarrollan habilidades y competencias clínicas, mejoran en su autoeficacia y aprenden a gestionar autónomamente 
su actuar según los estándares de la profesión. En este mismo sentido, también es preciso identificar e implementar indicadores observables susceptibles de triangulación metodológica para el establecimiento de los cambios que los propios supervisores experimentan gracias a la supervisión y que se relacionan, fundamentalmente, con el mantenimiento de un conocimiento disciplinar actualizado, y con un enriquecimiento y desarrollo de sus estrategias formativas como supervisores.

Todas estas contraposiciones y particularidades en el modelo lógico visionado por los supervisores de los programas de pregrado y maestría de la universidad participante permiten vislumbrar el camino investigativo por recorrer. Muy importante es el reconocimiento de la escasez de antecedentes de evaluación educativa de la supervisión clínica desde una visión programática. Así mismo, en consideración de la variedad de posturas epistemológicas relacionadas con la investigación en psicología, es posible argumentar que este estudio está limitado en términos muestrales y en su potencial para producir conocimiento generalizable. No obstante, dada la naturaleza hermenéutico-interpretativa de este estudio y sus métodos, así como su carácter educativo y evaluativo, el propósito principal no yace en la producción de conocimiento generalizable, sino de conocimiento contextualmente sensible (Stake, 1998, 2006, 2010) y útil para los actores primarios de los programas (Bryson, Patton \& Bowman, 2011).

En congruencia con esta postura, las necesidades inmediatas de esta investigación no llaman a reproducir este estudio o a ampliar o incorporar muestras comparables, etc., sino a desarrollar una comprensión profunda del funcionamiento pedagógico que se formule de estos programas, y más que en términos de concepciones, que incluya el contraste entre el funcionamiento percibido por cada grupo de actores con otros elementos del contexto y con la observación directa de su implementación y de sus resultados. De esta manera, será posible diseñar una juiciosa evaluación educativa con la cual establecer una valoración informada acerca de cómo estos programas, aparte de contribuir al logro de resultados educativos, tienen un impacto alineado con los mejores estándares de la profesión y, finalmente, cómo contribuyen favorablemente a la hora de proveer a los estudiantes una experiencia con cualidades y calidades meritorias y de valor.

\section{Referencias}

Alvim-Saraiva, L. \& Tiellet-Nunes, M. L. (2007). A supervisão na formação no analista e do psicoterapeuta psicanalítico. Estudos de Psicologia, 12(3), 259-268.

Association of State and Provincial Psychology Boards. (1998). Final report of the ASPPB Task Force on Supervision Guidelines. Montgomery, AL: Autor.

Auerbach, C. F. \& Silverstein, L. B. (2003). Qualitative data: An introduction to coding and analysis. Nueva York, NY: New York University.

Baird, B. N. (2011). The internship, practicum, and field placement handbook. Boston, MA: Prentice Hall.

Bernard, J. M. \& Goodyear, R. K. (2004). Fundamentals of clinical supervision. Boston, MA: Pearson.

Boris, G. D. J. B. (2008). Versões de sentido: um instrumento fenomenológico-existencial para a supervisão de psicoterapeutas iniciantes. Psicologia Clínica do Rio de Janeiro, 20(1), 165-180.

Bryson, J. M., Patton, M. Q. \& Bowman, R. A. (2011). Working with evaluation stakeholders: A rationale, step-wise approach and toolkit. Evaluation and Program Planning, 34, 1-12.

Castañeda-Cantillo, A. E., Abreo-Ortiz, A. M. \& Parra-Benavides, F. (2006). Aproximaciones a la construcción de un modelo de formación por competencias como interventores de sistemas humanos para estudiantes de psicología de último año. Diversitas: Perspectivas en Psicología, 2(1), 20-41. 
Cruz-Fernández, J. P. (2009). Enfoque estratégico y formación de terapeutas. Terapia Psicológica, 27(1), 129-142.

Falender, C. \& Shafranske, E. P. (2004). Clinical supervision: A competency-based approach. Washington, DC: APA.

Fortunato-Costa, L., Ribeiro, M. A., Penso, M. A. \& Campos de Almeida, T. M. (2008). O desafio da supervisão e pesquisa-ação em casos de abuso sexual: os profesores e suas questoes. Paidéia, 18(40), 355-370.

Friese, S. (2012). Qualitative data analysis with ATLAS.ti. Londres: Sage.

Goodyear, R. K., \& Nelson, M. L. (1997). The major formats of psychotherapy supervision. En C. E. Watkins (Ed.), Handbook of psychotherapy supervision. Nueva York, NY: Wiley.

Lizzio, A., Wilson, K. \& Que, J. (2009). Relationship dimensions in the professional supervision of psychology graduates: Supervisee perceptions of processes and outcome. Studies in Continuing Education, 31(2), 127-140.

Mafacioli da Silva, G., Colomé-Beck, C. L., Costa de Figueiredo, A. C. \& Cassol-Prestes, F. (2012). O processo do trabalho na supervisão clínico-institucional nos centros de atenção psicossocial CAPS. Revista Latinoamericana de Psicopatología Fundamental, 15(2), 309-322.

Malloy, K. A., Dobbins, J. E., Ducheny, K. \& Winfrey, L. L. (2010). The management and supervision competency: current and future directions. En M. B. Kenkel \& R. L. Peterson (Eds.), Competency-based supervision for professional psychology. Washington, DC: APA.

McDavid, J. C. \& Hawthorn, L. R. L. (2006). Program evaluation \& performance measurement: An introduction to practice. Thousand Oaks, CA: Sage.

McLaughlin, J. A. \& Jordan, G. B. (2004). Using logic models. En J. S. Wholey, H. P. Hatry \& K. E. Newcomer (Eds.), Handbook of practical program evaluation (pp. 7-32). San Francisco, CA: Jossey-Bass.
Milne, D. (2009). Evidence-based clinical supervision: Principles and practice. Malden, MA: BPS Blackwell.

Moon, J. A. (2004). A handbook of reflective and experiential learning: Theory and practice. Londres: RoutledgeFalmer.

Morris, S. B. \& Haas, L. J. (1984). Evaluating undergraduate field placements: An empirical approach. Teaching of Psychology, 11(3), 166168.

Ocampo, M., Suárez, R., Fonseca, M. A., \& Aguirre, D. C. (2012). Características socio-demográficas del psicólogo colombiano. En D. Castrillón-Moreno (Comp.), Condiciones sociodemográficas, educativas, laborales y salariales del psicólogo colombiano. Bogotá: Colegio Colombiano de Psicólogos.

Perkins, D. (1998). What is understanding? En M. Stone Wiske (Ed.), Teaching for understanding. San Francisco, CA: Jossey-Bass.

Rodríguez, D. M. \& Niño-Rojas, J. A. (2006). Marco comprensivo para el estudio de los procesos de formación de terapeutas. Diversitas: Perspectivas en Psicología, 2(1), 42-54.

Russell, R. K. \& Petrie, T. (1994). Issues in training effective supervisors. Applied and Preventive Psychology, 3, 27-42.

Stake, R. E. (1998). Investigación con estudio de casos. Madrid: Morata.

Stake, R. E. (2006). Multiple case study analysis. Nueva York, NY: Guilford.

Stake, R. E. (2010). Qualitative research: Studying how things work. Nueva York, NY: Guilford.

Stufflebeam, D. L. \& Shinkfield, A. J. (1987). Evaluación sistemática: guía, teoría y práctica. Barcelona: Paidós.

Stufflebeam, D. L., \& Shinkfield, A. J. (2007). Evaluation theory, models, and applications. San Francisco, CA: Jossey-Bass.

Teles-Tavora, M. (2002). Um modelo de supervisão clínica na formação do estudante de psicologia: a experiência da UFC. Psicologia em Estudo, 7(1), 121-130. 
Universidad de los Andes. (2008). Documento maestro del Programa de Maestría en Psicología Clínica y de la Salud. Bogotá: Autor.

Universidad de los Andes. (2014). Pregrado en psicología. Recuperado de http://psicologia.uniandes. edu.co/index.php/programas/pregrado-en-psicologia/por-que-hacer-el-pregrado-en-psicologia

Watkins, C. E. (1997). Handbook of psychotherapy supervision. Nueva York, NY: Wiley.
Wolfgang, D. G. (1976). The psychology teacher and undergraduate field experience courses: the problem of supervision. Teaching of Psychology, 3(4), 183-184.

Zaslavsky, J., Tiellet-Nunes, M. L. \& Laks-Eizirik, C. (2003). A supervisão psicanalítica: revisão e uma proposta de sistematização. Revista de Psiquiatria do Rio Grande do Sul, 25(2), 297-309.
Recibido: 8 de septiembre de 2014

Aprobado: 30 de junio de 2015 